\title{
Legitimación y funciones de la biblioteca en el contexto de la sociedad digital
}

\author{
José A. Gómez Hernández
}

Universidad de Murcia

\subsection{Resumen}

Estudio de la legitimidad de las bibliotecas en la sociedad de la información, revisando sus funciones y perspectivas desde el punto de vista de la incorporación de tecnologías, las posibilidades de la telemática, y las necesidades de información de los ciudadanos europeos. (Autor)

Palabras clave: Bibliotecas públicas. Funciones de las bibliotecas. Necesidades de los usuarios. Sociedad de la información.

\subsection{Abstract}

Review of the roles of libraries in the Information Age, considering the information technologies, the opportunities of using telematics, and the information needs of european citizens. (Author)

Keywords: Public libraries. Libraries roles. User needs. Information society.

\section{Introducción}

El objetivo de la reflexión que sigue es señalar algunas funciones de las bibliotecas en la sociedad en que nos encontramos o se nos avecina. La especial vinculación de la biblioteca con los libros, y la pérdida de éstos de su privilegiada situación como vehículos por excelencia de la cultura y el conocimiento, nos fuerzan a legitimar de nuevo la institución bibliotecaria, explicitando su utilidad social. Los que estamos persuadidos de que las bibliotecas, como espacio de aprendizaje, cultura, ciencia y comunicación siguen teniendo su lugar, debemos demostrarlo, evitando caer en tópicos que ya no se sostienen por sí mismos en un entorno de gran competitividad de recursos y servicios de información, y superando formulaciones voluntaristas o retóricas. La falta de argumentos facilita la extensión de tópicos sobre las bibliotecas, perjudicando su imagen, su reconocimiento social; y, de resultas de ello, su financiación. 
El objetivo es mostrar la potencialidad de las bibliotecas como medio idóneo para posibilitar la entrada en la sociedad de la información a la generalidad de los ciudadanos, especialmente a los que menos recursos tienen para utilizar los contenidos existentes, lo que daría a la biblioteca un protagonismo básico como servicio social. En Europa, las más de 40.000 bibliotecas públicas deben ser un recurso capaz de dar acceso a la información a los ciudadanos, y guiarlos en el proceso de analizar y aplicar esa información en su actividad privada y profesional.

\section{Las amenazas para las bibliotecas}

Podríamos preguntarnos si realmente las bibliotecas están en cuestión, si no estamos adoptando un enfoque apocalíptico o catastrofista. Lo cierto es que durante los últimos años se han construido hermosos edificios bibliotecarios, especialmente en las Universidades y en muchas ciudades. En las universidades sigue habiendo debates sobre las bibliotecas o la centralización de colecciones y algunos se niegan a ceder la gestión de sus bibliotecas a servicios centrales. La producción documental de las editoriales se mantiene e incluso crece en muchos sectores. Los maestros reivindican la biblioteca escolar (actualmente unos tres mil profesores siguen un curso sobre organización y dinamización de bibliotecas escolares basado en la tutoría telemática e Internet), etcétera. Viendo, por ejemplo, algunas bibliotecas de las grandes universidades, sus salas de ordenadores en red para la formación en tecnologías y productos de información, su mobiliario y su señalización o ante hechos como la contratación de algunos bibliotecarios para cursos universitarios, no parece justificada una visión pesimista sobre la realidad bibliotecaria actual.

Sin embargo, creemos que las amenazas están muy presentes para las bibliotecas que no están adaptándose con la velocidad suficiente a los cambios socioculturales, o cuyas actuaciones no tienen la necesaria repercusión social. Sólo la anticipación de los problemas podrá evitar que se vean desbordadas. Principalmente entiendo que la biblioteca como servicio de información estará en peligro mientras se mantenga pasiva ante las necesidades sociales de información y conocimiento, o cuando se concentre preferente o casi exclusivamente en un papel intermediario en los procesos de información de — si acaso- un grupo reducido de individuos. Los bibliotecarios que solo aspiren a esa función intermediaria, posiblemente no lograrán mantener la influencia y utilidad de sus servicios, porque cada vez son más amplias las posibilidades de acceso y consumo directo de la información.

Describiré mi experiencia personal para situar el problema, partiendo de que siempre he valorado las bibliotecas, y me he identificado con ellas. Así, siempre he considerado mi biblioteca como uno de mis patrimonios más queridos. He tra-

Scire. 4 : 2 (jul.-dic. 1998) 63-77. 
bajado como bibliotecario, y para mí la biblioteca ha sido una institución representativa de lo mejor de los hombres, de su razón, su creatividad, su historia, su vivencia. Además, la lectura de libros sigue siendo una de las experiencias que más me aporta. Sin embargo, he ido cambiando mis percepciones y prácticas en relación con las bibliotecas, de modo que actualmente, por ejemplo, como profesor universitario uso cada vez menos la biblioteca de mi Universidad, y la mayor parte de las veces no accedo físicamente a ella. El correo electrónico es una buena fuente de información personal e institucional, utilizo Internet para acceder a documentos muy recientes que la biblioteca aun no posee y de los que puedo obtener casi instantáneamente un ejemplar. El acceso y la comunicación de información por vía telemática ocupa una gran parte de mi tiempo de estudio e investigación. Incluso en la docencia utilizo con gran frecuencia ejemplos y casos a los que no he accedido por la biblioteca. Muchas de las veces que tomo libros en préstamo lo hago sólo para enseñarlos en clase o aconsejarlos por su carácter introductorio. De hecho, recomiendo menos la adquisición de libros a la Biblioteca, pues además de que a ésta le queda menos presupuesto para gastar en libros, estoy menos al tanto de conocer lo que se publica, leo menos libros y, por tanto, los aconsejo menos. A pesar de estar en plena sintonía con la idea de la biblioteca, cada vez yo mismo la uso menos.

Creo que uso poco las bibliotecas de mi entorno porque han sido poco activas frente a mis necesidades: me han estado proporcionando boletines de sumarios en papel cuando ya había tenido referencia electrónica de los artículos de mi interés, han hecho unos catálogos superficiales desde el punto de vista de la recuperación, no han vaciado las publicaciones periódicas o las compilaciones y actas de congresos, no me han permitido reclamar y obtener servicios desde mi ordenador (según algunos bibliotecarios tanto los alumnos como los profesores universitarios son demasiado cómodos) ni me han sabido resolver las demandas de información auténticamente complejas. La biblioteca no se ha preocupado de conseguir una buena colección de documentos electrónicos (solo unos pocos $\mathrm{CD}$-Rom y algunos enlaces a paginas web). Incluso a veces desaprovecho algunos artículos de revistas que me fotocopian, porque no tengo tiempo suficiente para leerlos, mientras ando por la red obteniendo información.

En otros entornos estoy percibiendo que muchos colegios están integrando las tecnologías de la información como instrumento didáctico saltando por encima o al margen de la biblioteca escolar, que sigue en su situación de marginalidad en la mayor parte de los centros, salvo cuando sale adelante por el voluntarismo de algunos maestros con vocación. Los niños de mis amigos no suelen acudir a las bibliotecas o dejan de ir cuando se hacen un poco mayores o autónomos. Muchas bibliotecas públicas están llenas, sí, pero de niños que los bibliotecarios luchan por controlar, y por estudiantes de apuntes que a veces transcriben

Scire. 4 : 2 (jul.-dic. 1998) 63-77. 
trozos de libros para trabajos de curso con los que no aprenden casi nada. Para una mayoría social muy amplia (recordemos que sólo el 11\% de los españoles acude al menos una vez al año a una biblioteca), el uso de las bibliotecas no se plantea: es un sitio poco atractivo, que quizás no tenga lo que yo puedo necesitar, o me ponga dificultades para usarlo, o no se adapta a mis requerimientos de consulta cómoda. Debemos reconocer que en general hay una gran mayoría de no usuarios de las bibliotecas: los profesionales, las clases medias trabajadoras, las amas de casa, los jóvenes, los ciudadanos adultos de ámbitos rurales, los ancianos; y, por supuesto, los que sufren cualquier tipo de marginación social, no acceden a la biblioteca, no la incluyen entre sus opciones cuando tienen un problema información o un deseo de tipo cultural o educativo.

Mientras que en las bibliotecas públicas siguen siendo predominantes los libros, veo a la gente comprar en quioscos siempre rebosantes y cercanos, enciclopedias en CD-ROM a precios mínimos, videos, discos, prensa y revistas, juegos, programas educativos, cursos de idiomas, literatura, teatro, historia o pornografía en todos los soportes. La información se vende y se consume directamente, pocos van a la biblioteca buscando bases de datos en CD-ROM en préstamo, pues se prefiere tenerlas y son económicamente accesibles, igual que la música, los programas, los videojuegos, las revistas, etcétera. Se ha generalizado para una parte de la clase media urbana un hábito de adquisición y consumo cómodo, en el punto de venta que hay debajo de casa, que se completa con la información que facilitan la televisión, la radio, el vídeo, la prensa o Internet, excluyéndose inconscientemente la biblioteca como recurso informativo. Y cuando se plantea un problema de información, o se resuelve de modo autónomo, a veces mal, o se consulta a un experto, no a un bibliotecario. El resultado es el agrandamiento de la diferencia entre una minoría consumidora directa de productos de información multimedia (sin mucho criterio, pues se compran revistas de informática porque regalan el Trivial en $\mathrm{CD}$, o una revista de actualidad política porque lleva un curso de inglés gratis), y el resto de las personas, cuya fuente básica de información son la televisión y las tertulias radiofónicas.

En este contexto la biblioteca como centro local de información es ignorada por muchos ciudadanos, y los que la usan no lo hacen en toda su plenitud. El entorno de telecomunicaciones tan diverso y caótico mantiene a las bibliotecas en una situación de incertidumbre, cuando a la vez existe una proporción mayor de información electrónica gratuita y accesible autónomamente por los enganchados a la red.

A esto se une, finalmente, que el uso generalizado y demagógico de la idea de progreso para legitimar los nuevos servicios y posibilidades de las tecnologías de la información hace que muchos vinculen la biblioteca con el origen ya desfasado, lo antiguo y viejo, el material bibliográfico obsoleto, frente a la unidad de 
información moderna, dinámica, instantánea, a la altura de su tiempo, culminación de la técnica, que ya no sería biblioteca. Cuando esta imagen, además de estar entre los tópicos de muchos ciudadanos, es asumida, creída o pregonada por el propio sector profesional o docente, evidentemente estamos perjudicando gravemente a la biblioteca: tenemos que ayudar a cambiar a la biblioteca para que cumpla sus fines, no arrumbarla para que no se nos pueda tildar de antiguos. El bibliotecario debe adaptar su ejercicio profesional a las necesidades y recursos disponibles y perseguir la calidad de su servicio, no abominar de su profesión renunciando a su nombre por otro que se atribuya un mayor componente de modernidad.

\section{Lo que se espera de las bibliotecas y los bibliotecarios}

Siento decir que ante ese panorama complejo algunos bibliotecarios se inhiben (al fin y al cabo tienen un trabajo agradable), no se implican lo suficiente, sienten impotencia o se conforman. Hay mucho por hacer, pero los bibliotecarios tienen que adoptar el compromiso de un ejercicio profesional basado en la búsqueda de excelencia en los servicios, en la utilidad social, en la investigación de los problemas de información de los usuarios, y en adaptar las modalidades de prestación de servicios a las posibilidades y necesidades de los ciudadanos. Y para ello, además, tiene que tener habilidad para superar obstáculos externos, como la insuficiente conciencia política y la endémica financiación deficiente, e internos, como la falta de formación profesional y la rigidez de posturas (1).

Cuando existe la posibilidad de acceder a casi cualquier información desde cualquier lugar, cuando la cultura registrada en cualquier soporte puede conseguirse por diversos medios, la biblioteca es la institución pública responsable de hacer posible y real el acceso a los que no pueden llegar por sí mismos a las fuentes. Es la base para atender necesidades de orden social de gran importancia, lo que la convierte en un servicio que no es solo cultural o educativo, sino de asistencia social en el sentido fuerte del término. Por eso se la relaciona con la implantación local de la sociedad de la información, con el mantenimiento de la democracia, la educación, la interculturalidad o la tolerancia, y le atribuimos estas funciones (2):

- Ser un espacio acogedor, un lugar de permanencia, de estancia, convivencia, comunicación, sociabilidad, que posibilite la reunión o encuentro de colectivos, personas diversas, etc. La biblioteca representa y debe ayudar a consolidar la convivencia, la tolerancia, el diálogo y la interculturalidad, un objetivo prioritario en una Europa cada día más mestiza y a la vez fragmentada. La biblioteca como institución cultural abierta es un lugar para el intercambio, la diversidad, y la enseñanza de la convivencia y la tolerancia. Debe hacerse habitual un uso solidario, compartido y simultáneo de recur- 
sos comunes y diversos, tanto para la integración cultural como para el mantenimiento de las raíces y tendencias propias, lo minoritario o lo que sale de la norma. La biblioteca como espacio, plaza pública, ayuda a mejorar la oferta cultural de la ciudad, haciéndola más habitable y viva. Su oferta de espacio es una de las ventajas de las bibliotecas: No toda la convivencia se puede dar por correo electrónico o videoconferencia, la comunicación interpersonal tiene que ser también física, de contacto, corporal, y la biblioteca permite cultivar la cultura en común. A los usuarios les gusta usar la biblioteca no solo para obtener información sino como espacio de lectura, trabajo, encuentro, callejeo, curiosidad..., como espacio de sociabilidad. La biblioteca debe ser para ellos un espacio no segregador, en el que fomenten o posibiliten prácticas culturales diversas, en un marco elástico y maleable, no directivo (Cencerrado, 1997).

- Proporcionar acceso (mediante consulta, préstamo o reproducción), con espíritu democrático, a toda la información que se publique, haciendo posible el acceso a los documentos en cualquier soporte y electrónicos. No es posible siempre la adquisición ni la disponibilidad de todo lo difundido salvo a través de la biblioteca, luego ésta es imprescindible para hacer efectivo el derecho a la información de los que tienen poca capacidad adquisitiva. La biblioteca debe ayudar a relanzar la información, ser un punto facilitador y multiplicador de los accesos, organizándolos y haciéndolos efectivos. Debe tener una colección amplia, diversa, equilibrada y viva. Frente a la librería o el quiosco como supermercados de productos del día, la biblioteca tiene la ventaja de su aportación de recursos documentales complementarios.

- Colaborar con el sistema educativo, con los centros escolares, y ofrecer oportunidades de educación permanente, tutorizada o independiente. La biblioteca es un medio más de formación autónomo y no reglado, complementario del resto de la oferta formativa profesional y personal existente. La educación por medios telemáticos, que se extenderá en la sociedad digital, necesita apoyos, tutoría, medios complementarios, que la biblioteca debe proporcionar. La biblioteca debe apoyar o incluso iniciar desde programas educativos de alfabetización (3) los planes de reciclaje profesional.

- Hacer posible que los ciudadanos usen ordenadores y tengan acceso al equipo y a los sistemas que necesiten. En este sentido, la disponibilidad de las tecnologías de información en las bibliotecas es un apoyo importante para compensar las desigualdades económicas que impiden todavía a muchos ciudadanos familiarizarse y trabajar con herramientas ofimáticas (4). La biblioteca debe dar acceso a redes, y ayudar en el proceso de su uso. La biblioteca pública debe ejercer como centro local de acceso al hardwa- 
re, al soporte lógico y a las redes, para que los ciudadanos puedan utilizar tecnologías nuevas y de amplia aplicación, favoreciendo una nueva alfabetización, la que permite usar las tecnologías para el mejor manejo de la información. Las bibliotecas móviles también deben acercar estos recursos a los menores núcleos rurales (García Gómez, 1997), para compensar la falta de acceso a la información, limitada casi siempre a la recibida por televisión.

- Facilitar el acceso a las más diversas manifestaciones artísticas, e incluso posibilitar directamente actividades culturales y estéticas de sus ciudadanos. La biblioteca como centro público debe atender a la diversidad: los gustos literarios de los jóvenes, de los ancianos, los cómics, la música culta y la popular, la poesía última y minoritaria así como los materiales populares más vendidos. Los centros culturales pueden ser focos de atracción y reunión de los ciudadanos, y las bibliotecas deben formar parte de esa oferta cultural de las ciudades que las haga atractivas tanto para residentes como para visitantes.

- Conservar el pasado, la cultura, la historia, las producciones intelectuales de los hombres, permitiéndonos comunicarnos en el espacio y el tiempo, gracias a la preservación, clasificación y conservación de los documentos, para que lo lean por sí mismos los que lo deseen.

- Salvaguardar la identidad cultural en un mundo en rápida evolución. Las bibliotecas públicas son un foco de fijación, conservación y acceso a la cultura propia, local, complementaria a la información que llega a la aldea global. La colección de los autores locales, la documentación sobre éstos, los recitales de sus obras, los documentos orales, las revistas de grupos locales, las tradiciones folklóricas, deben tener su registro en la biblioteca. La biblioteca debe fomentar la literatura en su ámbito, el conocimiento del patrimonio cultural propio, la preservación de la tradición oral, y para ello puede fácilmente producir contenidos y difundirlos convencionalmente y por las redes.

- Proporcionar asesoramiento, orientación, e información para y sobre la comunidad elaborada por la propia biblioteca, ayudando a fomentar conocimientos y valores, la generación de actividad cultural, etc. La biblioteca debe garantizar a los ciudadanos el acceso a todo tipo de informaciones de la comunidad: servicios, organizaciones y asuntos concernientes a la localidad.

- Proporcionar servicios especiales a diversos grupos de usuarios, desde información empresarial hasta servicios para minorías étnicas, inmigrantes (González, 1997) y personas con deficiencias, situaciones de marginación 
o dificultades de integración: enfermos, reclusos, parados, ancianos impedidos para desplazarse, discapacitados sensoriales, psíquicos y físicos, drogadictos, etc. La biblioteca es una institución compensatoria de las desigualdades crecientes entre ricos y pobres en información, y la tecnología un apoyo para muchos de los colectivos desfavorecidos.

- Crear, apoyar y consolidar los hábitos de lectura. La lectura es un medio de placer y estímulo de la imaginación y la creatividad en los niños y jóvenes desde los primeros años. La lectura enriquece la vida propia a través de la comunión con otras vidas que se produce en los textos literarios. Amplia la propia experiencia aportando el conocimiento de otras realidades sociales. Amplía el lenguaje y ayuda a aprender. Ayuda al encuentro del sí mismo y favorece la formación de una capacidad crítica ... Dar a leer los textos, múltiples y diversos, favorecer y ayudar a elegir es otra función de la biblioteca, un espacio en que se debe propiciar el encuentro con la poesía, el teatro, la narrativa, ayudar a la formación del propio gusto literario, etc.

- Ser centro de actividades locales: La biblioteca es un punto central clave de las actividades, reuniones y servicios de la comunidad, especialmente en los ámbitos municipales donde los espacios alternativos suelen ser bares y poco más. Los grupos juveniles de música, las asociaciones de barrio, los colectivos sociales deben poder aprovechar los espacios bibliotecarios.

- Ayudar a la gente a adaptarse a la nueva situación de información, educando las habilidades y estrategias para encontrar la información, discriminar la relevante, comprenderla, organizarla e aplicarla para dar solución a sus problemas de información. Fomentar una cultura de la información (Cornella, 1997), procurar el aprendizaje de las fuentes de información y las habilidades para utilizarlas permite el desarrollo de la autonomía personal, y hace la biblioteca por lo tanto un espacio de democratización, de formación de los criterios personales. El personal bibliotecario al realizar esta función da a la biblioteca la posibilidad de diferenciarse de la librería o los supermercados. Es importante preparar para el acceso electrónico, facilitarlo, reducir los problemas que se dan todavía en él (5), de modo que los que en principio se sienten reacios o escépticos no pierdan la oportunidad de aprovechar sus beneficios.

- Atender, alentar y respetar la diversidad, permitiendo la diferencia, la transgresión, la ofensa, contra toda censura, formando una colección dotada también de lo minoritario.

Cuantificar lo que aportan las bibliotecas al realizar estas funciones es difícil, pero el valor añadido de la información que producen y difunden es enorme, porque la biblioteca posibilita la generación de ideas productivas, la educación de 
las personas, y la integración de los ciudadanos en situación de riesgo de marginación, haciendo la sociedad mejor. Debemos conseguir hacer reales estas aportaciones posibles de las bibliotecas, para que se evidencien las ventajas de su buena financiación, y captemos el amplio y heterogéneo colectivo de usuarios que ni sienten como carencia su falta de información, ni creen que la biblioteca les pueda servir.

\section{4. ¿Cómo y para qué han incorporado las bibliotecas las tecnologí- as de la información?}

Las bibliotecas han ido introduciendo las tecnologías de la información con dificultades, porque implican cambios y decisiones que resulta difícil tomar, asumir, financiar y ejecutar. El proceso de incorporación no siempre ha seguido un plan definido, sino que se ha actuado a remolque de las circunstancias, resultando estas etapas:

- Automatización del catálogo, que en muchas bibliotecas aún no se ha completado, por la amplitud de sus colecciones. En ese momento no se aplicaba la automatización para mejorar otros servicios o la información sobre la propia gestión, sino como una nueva herramienta para el proceso técnico.

- Acceso a bases de datos en línea para personal y usuarios. Numerosas bibliotecas, especialmente las universitarias y científicas, disponen de redes locales de consulta de bases de datos en CD-Rom.

- Incorporación a una red local, usada para intercambio de información por los profesionales, correo electrónico, etc. Así lo han hecho ya la generalidad de las bibliotecas universitarias españolas, por ejemplo.

En la actualidad, las bibliotecas más avanzadas han ido incorporando Internet para obtener información externa; $\mathrm{y}$, a su vez, van creando poco a poco páginas web propias. Al principio como ventana de presentación de los servicios, acceso al catálogo y a las normas de uso; y, poco a poco, algunas bibliotecas van prestando servicios de modo interactivo a través de la red. La entrega electrónica de documentos, la gestión de préstamo interbibliotecario por correo electrónico, la respuesta a demandas informativas por esa misma vía, la atención de desideratas o sugerencias, están empezando a darse. Y se está comenzando a plantear como problema por algunas bibliotecas universitarias y científicas la prestación de documentos conocidos por usuarios remotos y ajenos al sistema bibliotecario local que los conocen y piden a través de la página web.

Estos pasos no han sido dados por todas las bibliotecas. En el último estadio descrito se encuentran muchas bibliotecas universitarias y científicas, que han crecido para atender necesidades concretas de estudio e investigación. También, las bibliotecas regionales, que han encontrado contextos de autoafirmación auto- 
nómica muy favorables, y las de grandes capitales. En cambio, la gran mayoría de las bibliotecas de base, públicas municipales y escolares, están en precario, tanto por sus recursos como por sus servicios. La masa de bibliotecas municipales de ciudades medianas y pequeñas están todavía en situación de atraso, si acaso empezando la automatización de los catálogos, y fuera de la Red, de la biblioteca virtual de ámbito universal. Esta mayoría es la que debería, por su cercanía a los ciudadanos con menos recursos e infraestructuras culturales y educativas, protagonizar el empuje de la sociedad de la información en Europa.

\section{La nueva biblioteca y el nuevo bibliotecario}

El web ha traído consigo un incremento enorme del acceso en línea a publicaciones completas e información de múltiples tipos, cada vez usada por más personas. Las bibliotecas, aunque sigan dando acceso a los documentos impresos, muchos de los cuales tendrán que seguir adquiriendo (6), deberán dar acceso a materiales que no sean de su propiedad, y sobre todo cada vez deberán proporcionar más recursos electrónicos, realizando sobre ellos tareas técnicas parecidas a las tradicionales.

En cuanto a los materiales impresos y la posibilidad de su desaparición en su forma actual, un aspecto muy importante para el modo de funcionamiento de las bibliotecas, nos parece especialmente razonable lo observado por Line (1997). Para éste, dentro de unos diez años la mayoría de los libros se publicarán como hasta ahora, con la excepción de obras académicas con un mercado reducido, que se publicarían bajo demanda. Igualmente, la mayoría de las revistas y periódicos para el público en general se publicarán como en la actualidad. En cambio, la mayoría de las revistas científicas fundamentales, aunque tengan una versión en papel, también serán accesibles en línea y posiblemente en formato electrónico tangible (especialmente para la colección retrospectiva). Y la mayoría de las revistas científicas marginales serán accesibles únicamente en línea.

En esta perspectiva, algunas tareas de intermediación tradicionalmente bibliotecarias pueden verse en crisis porque se oferten directamente a los usuarios, transformando el mercado de la información: servicios de distribución electrónica de información científica, acceso a publicaciones en línea de modo gratuito junto a la suscripción impresa, y obtención directa de documentos alojados por los autores en sus webs.

El acceso directo de los usuarios finales a los recursos de información distribuidos por el web competirá cada vez más con los servicios convencionales de las bibliotecas. Aunque esto no las reemplazará, incrementarán su importancia si son capaces de adaptarse a nuevas responsabilidades y oportunidades, incrementando sus servicios y contenidos dados por la red. Ello las obligará a (7): 
- Desarrollar pautas de acceso a colecciones remotas, que no siempre serán gratuitas y requerirán controles de acceso según grupos y tipos de usuarios, con diversas condiciones económicas. Para el usuario remoto no existen las barreras geográficas; por tanto, el centro habrá de atender "virtualmente" a usuarios "virtuales", de forma muy interactiva.

- Crear, incorporar, gestionar y hacer que se usen colecciones electrónicas propias, cuya importancia crecerá conforme este medio sea el elegido para los materiales bibliotecarios y los documentos de todo tipo. Los bibliotecarios tendrán el reto de dotar de contenidos de interés a su web de modo que la biblioteca se use de modo remoto tanto o más que localmente.

- Conseguir dar acceso de modo integrado a información en todas sus formas, del modo más efectivo en relación con el coste.

- Adaptar recursos y prácticas de gestión a un entorno caracterizado por el cambio continuo, siendo capaces de diseñar, aplicar y evaluar soluciones para los problemas de información y de servicio presentados (Gómez 1998).

- Actualizar continuamente su infraestructura tecnológica, para mantenerse al día frente al cambio continuo en la gestión y uso de la información.

- Aprovechar al máximo la cooperación interbibliotecaria en redes locales, regionales y supranacionales.

- Intensificar la formación de usuarios para el acceso electrónico: En primer lugar, formación en técnicas de recuperación de información a través de la Red, manejo de operadores booleanos, mejora de las búsquedas y realización de búsquedas avanzadas. Pero, en segundo lugar, formación en el análisis y aprovechamiento de los contenidos obtenidos.

- Ofrecer documentos electrónicos propios de temas de especial relevancia para los usuarios de la biblioteca a través de Internet, de modo complementario a los grandes servicios internacionales o de las bibliotecas nacionales. Es decir, producir, mantener y difundir bibliotecas virtuales propiamente dichas.

La biblioteca debe saber combinar lo inmaterial, la información, con lo espacial, lo virtual con lo real, el encuentro con la información lejana, con la orientación presente e interpersonal. Lo cognitivo con lo afectivo, el saber con la lectura. La comunicación de información, con la comunicación afectiva. La biblioteca por lo tanto debe ser un espacio de comunicación que ayude a la gente a estar informada y tener criterios sobre su saber. La biblioteca deberá aportar a la vez una colección de recursos informativos en sentido amplio, con salas para consul- 
tas digitales adaptadas al uso de las tecnologías, junto a instalaciones culturales y propuestas sociales.

¿Podemos denominar biblioteca al servicio que integre las actuaciones descritas? En mi opinión, sí. Según hemos visto, la biblioteca es una unidad de información dotada de colecciones convencionales e impresas, que difunde junto a la prestación de otros servicios culturales, sociales y educativos. Mejor que darle otros términos (como mediateca, infoteca), lo que supone arrumbar la denominación que asumen las decenas de miles de bibliotecas existentes, es ayudar a su dinamización.

¿Y el bibliotecario de esta nueva biblioteca, debe cambiar su nombre? En mi opinión, no. El bibliotecario se integra, junto a otros profesionales, en el colectivo de los expertos en información, a los que podemos convenir en denominar de modo general documentalistas, infonomistas (según la propuesta un poco altisonante de Cornella), o simplemente informadores, gestores de información o ingenieros en información si se quiere resaltar el componente tecnológico.

Dentro de esta adscripción general al colectivo de profesionales de información, el bibliotecario tiene un perfil propio y específico, que integra habilidades de información, comunicación, gestión institucional y cultural, etc. Mejor que cambiar el nombre del que trabaja en la biblioteca, es hacer evolucionar su actuación profesional. El bibliotecario debe adaptar su actuación a los medios disponibles y a las necesidades sociales que hemos explicado, lo que le obliga a ejercer roles, competencias y habilidades complejas y distintas de las que eran básicas cuando el libro era la base de la identidad profesional del bibliotecario. Entre ellas están el dominio de técnicas de recuperación de información digital y la formación técnica de los usuarios en sala. Pero la diversificación de la biblioteca y su apertura al usuario lo obligan a ser gestor, investigador, animador cultural, diseñador de productos culturales, y sobre todo experto y capaz de colaborar en equipo para lograr una prestación óptima de los servicios demandados.

\section{Reflexiones finales}

Entre algunas recomendaciones que cabría señalar para que las bibliotecas cumplan su función en la sociedad de la información, señalaríamos las siguientes (8):

- Deberíamos fomentar el mayor protagonismo posible de la biblioteca dentro de la política cultural de las comunidades locales.

- Para aprovechar su potencial, las bibliotecas públicas deben dar servicios nuevos y ampliados de cara al ciudadano, tanto de carácter informativo como educativo y sociocultural. 
- Es necesario apoyar la formación profesional permanente de los bibliotecarios para que puedan seguir el ritmo de los cambios tecnológicos y las demandas informativas.

- Debería fomentarse una colaboración muy estrecha entre diversos tipos de biblioteca y otras instituciones y proveedores que ayuden a aportar contenidos y posibilidades al sistema bibliotecario.

- Ayudar al diseño de materiales multimedia que llenen de contenidos de interés las bibliotecas virtuales.

- También es muy necesario crear materiales educativos multimedia para la formación de usuarios.

La trascendencia de las funciones sociales de la biblioteca pública es enorme, y casi produce vértigo. Los profesionales debemos mantener un gran nivel de formación, autoexigencia y adaptación para lograr dar unos servicios de un nivel acorde con las demandas de la época. Al luchar por conseguirlo estaremos ayudando a legitimar de nuevos los servicios bibliotecarios, y contribuyendo a que salgan de la situación de marginalidad que en muchos lugares todavía mantienen.

\section{Notas}

(1) Así, el Informe Las bibliotecas públicas en la sociedad de la información (Thorhauge, 1997) señala que: "Como consecuencia de una educación pasada de moda o de la falta de formación permanente y de oportunidades de formación, el personal no posee la calificación suficiente. Es necesaria una formación profesional para el desarrollo a escala nacional, regional e institucional. Si falta tal estrategia es en gran parte consecuencia de unas capacidades de gestión inadecuadas, de la poca disposición para familiarizarse con los nuevos medios, del miedo al cambio, y de la escasez de nuevo personal con calificaciones actualizadas en TI". Los contenidos de la formación en tecnologías necesarios para los usuarios han sido recientemente estudiados en Pors (1996), recomendando especialmente la formación a través de medios telemáticos y el adiestramiento en el propio puesto de trabajo.

(2) Consideramos, entre otros, el Manifiesto de la Unesco, McClure (1991), Thorhauge (1997), y el texto 12 ways... (1995).

(3) El apoyo educativo a las familias y a las escuelas es especialmente destacable en las bibliotecas americanas, en las que hay centros para hacer los deberes, alfabetización de familias rurales, ayuda escolar a domicilio, programa de lectura estivales, servicios in situ a centros de acogida para indigentes, residencias de ancianos y guarderías. Unas seis mil bibliotecas públicas cuentan con un empleado que enseña a leer y escribir (12 ways..., 1995).

(4) La media de hogares con ordenador en España está en el 19,2\%, pero concentrándose especialmente en regiones ricas como Madrid, Cataluña y Navarra, y en las grandes ciudades, con una penetración menor en comunidades pobres como Extremadura o Castilla-La Mancha (9\% de hogares con ordenador) y zonas rurales. El ordenador 
está presente sobre todo en los hogares de profesionales de los servicios, mientras que es muy escaso en las zonas de actividad agropecuaria. Además, la mayor parte de estos ordenadores carece de CD-Rom, y en cuanto a conexión a Internet, estaba por término medio en un $2 \%$ de los hogares. Datos de Vacas, et al. (1998).

(5) Line (1997) observa dificultades en el acceso electrónico de usuarios cualificados como los científicos, detectando que hay muchas diferencias en el aprecio y aceptación de esta posibilidad, junto a problemas como una frecuente frustración y la pérdida de tiempo por la inadecuación del software de búsqueda, la ineficacia de la interfaz de usuario, y del volumen de material inútil. Además, el modo de pago, y que la información que se obtiene puede no ser la necesitada, hace que para bastantes científicos el papel sea todavía soporte preferido por algunos usuarios y para algunos propósitos.

(6) Line(1997) ha hecho hincapié, sin embargo, en que los materiales más necesitados se siguen teniendo que adquirir, para no dejar la biblioteca en situación precaria. Apoyarse en la accesibilidad y no en la propiedad genera una gran dependencia de otras bibliotecas. Además, no se sabe cuánto costará el acceso electrónico si acaba siendo la norma para las revistas científicas, ni tampoco las restricciones que se establecerán para su uso.

(7) Nos basamos principalmente en el documento de difusión del plan estratégico de OCLC para el año 2000 (OCLC 1997).

(8) Principalmente seguimos las propuestas en Thorhauge (1997).

\section{Referencias}

12 ways libraries are good for the country (1995). // American Libraries. (1995) 11121119. Citado por la trad. de Educación y Biblioteca, 86 (1998) 32-40, bajo el título Las bibliotecas...

Eicher. C. (1997). Bibliotecas públicas : la fuerza socioeconómica del entorno urbano. // La Biblioteca Pública, un compromiso político : I Jornadas Biblioteca Pública y Políticas Culturales. Barcelona : Fundación Bertelsmann, 1997.

Cencerrado Malmierca, L. M. (1997). La formación de usuarios como difusora de los servicios de la biblioteca. // Boletín de AABADOM. 8 : 2 (1997) 31-35.

Cornellá, A. (1997). ¿Economía de la información o Sociedad de la información?

García Gómez, J. C.; Martínez Méndez, F. J. ; Gómez Hernández, J. A. (1997). Bibliotecas digitales de Internet como servicio complementario para pequeñas bibliotecas. // 6es Jornades Catalanes de Documentació. Barcelona : Societat Catalana de Documentació i Informació ; Col-legi Oficial de Bibliotecaris-Documentalistes de Catalunya, 1997. p. 275-284.

Gómez Hernández, J. A. (1998). Un Espace pour la bibliotéconomie dans les sciences de la documentation : étatas des lieux en Espagne. // Bulletin des Bibliotheques de France. 2 (1998) 61-66.

González Quesada, A. (1997) La Biblioteca pública como uno de los protagonistas en la integración de la población inmigrante. // V Jornadas sobre Bibliotecas Infantiles, 
Fundación Germán Sánchez Ruipérez, Salamanca, 27-28 de junio de 1997. Copia de la conferencia.

Line, M. B. (1997). Electronic information : use and users. // 6es Jornades Catalanes de Documentació Barcelona : Societat Catalana de Documentació i Informació : Col-legi Oficial de Bibliotecaris-Documentalistes de Catalunya, 1997. P. 25-36. Versión castellana de: C. Urbano Salido en Anales de Documentación. 1 (1998) 198212.

Maclure, C. H. (1991). Manual de planificación para bibliotecas : Sistemas y procedimientos. Madrid : Fundación Germán Sánchez Ruipérez, 1991.

OCLC (1997). Beyond 2000 : A summary of OCLC`s Strategic Plan. Dublin ; Ohio : OCLC, 1997.

Pors, N. O. ; Screiber, T. (1996). Librarian Training in Information and Communication Technologies : A typology of Needs and Deliverables.

Thorhauge, G. ; Larsen, H. P. ; Alberchtsen, M. S. (1997). Public libraries and the Information Society. Luxemburgo : Oficina de Publicaciones Oficiales de las Comunidades Europeas, 1997.

Vacas Aguilar, F. ; Reyes Barragan, M. J. ; Castro Castro, C. (1998). Tecnologías de la información en los hogares de Extremadura. 